\title{
Falseness in the miRNA-field as an indicator of strategic bias in the research system via peer-review and publishing eligibility
}

\begin{abstract}
Bias is the main barrier to a current scientific understanding. It is the tendency which prevents unprejudiced consideration of a question, and the deviation of the expected value of the reality, and the systematic error introduced into any testing by selecting or encouraging one outcome over others. It is not only the systematic error introduced to samples-it is also the systematic error introduced in any system including systems of thought and entire research fields. The case of the miRNA (miR) research field helps to illustrate and resolve these inherent strategic biases in science that only benefit the biasing peers at the expense of everyone else. Genomics especially requires an unbiased assessment of the genome and the many gene functions in a collective, free but unbiased systems biology approach with equal and fair opportunity and enough jobs for PhDs and PostDocs as a relevant prerequisite. Among the many genes are also genomic microRNA loci that are processed into small non-coding but conserved double-stranded miRs. Until today, these miRs are thought to fine-tune mRNA and protein levels by degrading mRNAs or by blocking their translation and both. A closer look at this interesting class of small noncoding RNAs, however, reveals that there are not only canonical miRs and miR-seeds but also non-canonical forms, i.e. isomiRs and isoSeeds. Although canonical miRs and miR-seeds are easier to identify, the majority of miRs could be non-canonical depending on the cutoff of the definition. Senior researchers and peers have introduced strategic biases to omit the underlying complexity to make an impact, career, patents, and business, and have systematically hindered PostDocs and $\mathrm{PhDs}$ and their publications foryears.
\end{abstract}

Keywords: bias, strategic bias, mirna, isomer, isoseed, loci, mirbase, seed, sequence, research system, falseness, dysfunction, network, censorship, scientific, oppression, eligibility, publishing, peer-review
Volume 4 Issue 3 - 2017

\author{
Roman Anton \\ University of Truth and Common Sense, Germany
}

Correspondence: Roman Anton, University of Truth and Common Sense, International and Darmstadt, Mangoldweg 27, 64287 Darmstadt, Germany, Tel 49172914329I,

Email mail.roman.anton@gmail.com

Received: November 05, 2017 | Published: December 19, 2017
Abbreviations: Deep sequencing, ngs with a high number of replicate reads; GCR8, digeorge syndrome critical region 8 gene; isomiR, the variations of mirna sequences around the published mirna sequence at mirbase, which is misleadingly referred to as gold standard database-and due to this a major source of bias the author of this review claims; isomiRs, all similar miRs variations processed from the common genomic loci-there can be more than one seed per miR; IsoSeeds, all functional miR-seeds along the miR-or isomiRstrand that are matching with RNA or potentially DNA in canonical or non-canonical ways, via Watson-Crick or non-Watson-Crick basepairing; KO studies, knock-out studies; LOB, level of bias; miRNA, micrornas; miRISC, miRNA-induced silencing complex; miRs, microRNAs; NGS, next-generation sequencing; nt, nucleotide; OTV, overall truth value; qPCR, quantitative polymerase chain reaction; RPF, ribosome-protected mRNA fragment; RNA, ribonucleic acid; RVSA, the real value of scientific achievement; TNV, true novelty value

\section{Introduction}

The complexity and diversity of genomics on the nucleotide (nt) level further increase exponentially on the transcript and on the respective modification and functional level of genes, proteins, and RNAs, and their downstream catalytic and metabolic effects. Among the many different forms of genomic and epigenetic gene regulation whose publications have also increased partially exponentially, layer by layer, year after year, we find the role of small non-coding doublestranded RNAs. These microRNAs, miRNAs or miRs, of roughly 18$24 \mathrm{bp}$ in length, have attracted much attention of scientists and the life science industry. Today, miRNAs are still thought to regulate gene function at the transcript level by destabilizing mRNAs or by affecting the efficiency of their translation, or by an intricate interplay of both..$^{1,2}$ miRNAs are also defined as short double-stranded unique noncoding and regulatory RNA sequences. Moreover, a single miR-BASE entry widely serves as the major definition, one sequence with one miRidentity number that is believed to be "the real miRNA" and to exert these functions by imperfect base-pairing of somewhere once-defined "seed sequences". ${ }^{3}$ This review, instead suggests a role of isomiRs and isoSeeds and different miR-functions and widely different miRtargets. It also claims that academic genomic research and industry innovations were somewhat biased to focus mainly on single miRsequences and their targets, and mechanisms in the last decade.

The overseen diversity of canonical and non-canonical isomiRs and isoSeeds, different miR-functions, as well as the imperfect basepair matching and binding of miRs, has led to a guessing-bias, a don'tquestion-the-dogma-bias, a make-things-significant-bias, and a haveto-claim-as-first and first-publication-bias, among many others. ${ }^{4}$ Such publishing biases are furthered by a dominant semi-scientific peerpressure based peer-review publication system and its procedures 
and all mainstream-publishing demands and criteria. This silently forces authors to follow previously published stereotypes and pattern to achieve the right impact points via giving the right gut feeling in peers and only intends to give the 'right scientific impression' of an otherwise semi-scientific and often semi-false and only semi-new content, both visually and in reading. This is neither a critique of early hypothesis building nor a critique on preliminarity of science. It is a critique of forced semi-wrong mainstream publishing, peer and senior dominance and lack of normal opportunity for PhDs and PostDocs. It is a critique of peer and system bias and behavior that does not allow to question any pre-assumptions-and that mainly strives to only appear like very professional science but is not. Strategic bias has helped research explorers and exploiters to publish semi-correct results to meet a new often only fake success-driven semi-scientific publishing imperative that has taken over the entire scientific landscape in all fields and nobody did stop it like in a global Milgram-experiment in publishing that follows a misleading authority bias without feeling any own responsibility for the drama it causes for PhDs, Postdocs, the scientific truth and the future of all sciences.

Quality assurance by peer-review is generally too biased to ever function and unbiased publishing eligibility does not exist. Scientist peers have been indoctrinated, at least historically by previous and strategically by recent peer networks and participate in a by default semi-wrong semi-scientific and semi-honest publishing in an only for them beneficial swarm bias behavior. Every field thus behaves and benefits like a swarm that follows the trends and semi-false biases in proposition, research, outlook, and promise. By consequence, a mainstream default blockage of all new and alternative hypotheses, true and honest scientific findings and interpretation appears that simultaneously silences and inhibits PostDocs and PhDs, especially ambitious ones who tend to strive for a new breakthrough result, innovation, and scientific or a related business career. The absolute requirement or funding to propose semi-false grants is just one key example of it that is subsequently driving more bias everywhere. Concomitantly, semi-correct researching and publishing became the secret new network standard like arbitrary decision making. These essentials to be productive can now also be seen in all other fields of science-not only in the miRNA-field. The strategic bias in the miRfield is just a case example to first illustrate the major dysfunctions in the scientific systems worldwide that hamper fair career paths and options for all scientists, and the unbiased truth in science.

\section{Discussion}

Conflicts of interests and publishing imperatives are the key drivers of semi-false scientific judgment, however, they can be found more frequently everywhere in science of today. This has led to mainstream scientific publishing schemes, which more and more make authors follow the peer-review patterns in a mandatory form that only seems and feels intuitively right but it is semi-false, often not helpful or reproducible, key details and honesty are missing, and this is very much biasing the fields. Starting with the title " $\mathrm{X}$ mediated by $\mathrm{Y}$ is controlled by Z" or "W-induced X promotes Y by Z", or "X is somewhat linked to $\mathrm{Y}$ in $\mathrm{Z}$ ", and so on, and subsequently this seemingly standardized bioinformatics-like pattern of publication would now require several unambiguous 'hard facts' to meet expectations - but most of these publications cannot unequivocally deliver such results and too much want to do so and will finally give the impression as if they would. Even semi-false, falsely significant up to totally fake results that still could be the case but never were done or done this way "-to make the entire story work", on experiments, results or methods. Whoever sticks to the real-world wet lab facts has to drive the extra mile without the funding and time "fuel" available for it and loses the productivity needed to be successful in this world of bad sciences-and good science of PostDocs is everywhere stopped by publishing eligibility, peer bias, and by an extreme lack of enough faculty positions. ${ }^{5-9}$ PIs handover all of this pressure to do wrong or semi-right to junior scientists, especially PhDs and PostDocs to drive semi-correct publishing or no paper and out forever. These publication patterns of standardized falseness and standardized bias are increasingly found, increasingly misleading, and correlate with the strategic oppression of junior scientist. ${ }^{5-9}$ This has led to the big success of semi-correct publishing, scientific network robbery of intellectual property and of the ideas and projects of $\mathrm{PhDs}$ and PostDocs. It has given much incentives for 'bad PI behavior' and makes bad publishing much easier than good and correct or adequate scientific reporting: fully correct scientific reports are much more 'risk exposed' in a world of bad science and $\mathrm{PhD}$ and PostDoc repression due to their inherent honesty, clarity, realness of progress, and the uncredited advancement of methods and ideas. All of this is often not found today and high-impact point incentives of journals have worsened all even some more. Simplification is sometimes needed but if it follows a bias or stereotype it gets problematic.

High value but less appreciated scientific contributions, i.e. with a higher but widely undetermined RVSF (real value of scientific finding), with respect to progress, can seem often less striking, less eye-catching or less bold, or identify less optimally functioning mechanisms, more admittedly. The scientific field much aims at a new fake professionalism that only needs to seem professional or new. It does not need to deliver more nor does it have to be fully reproducible due to its grey field, which, however, has caused a game-theoretical trap for all scientists and sciences that we are all in already - not only in the miRNA field but also in literally every field of science probably. This critique would allow preliminary findings and does allow same patterns and new patterns, a storylike or story-less publishing, spartan or comprehensive interpretation. But it would forbid eligibility and peer-review publishing and the recent artificial research and career scarcities strategically placed by strategy consultancies for PhDs, PostDocs and especially senior PostDocs that altogether always compels a research success at the cost of the common welfare and the Good Sciences and the quality of the scientific truth, which will be still needed to build on any of the previous results in the future to make progress. It would also allow junior researchers, $\mathrm{PhDs}$, and PostDocs, to publish without all of these blockades and hindrances and would ensure sustainable career paths for all scientists. The miRNAfield exemplifies the inherent bias in science and the problematic developments for scientists.

In the miRNA-Field, introductions in miRNA papers have tended to start with: miRNAs were first discovered in C. elegans in $1993^{10}$ as a regulatory class of evolutionarily conserved small non-coding $\mathrm{RNAs}^{11}$ that block transcript stability and translation via imperfect miRNA-mRNA base-pairing and miRISC. ${ }^{2}$ Such text rituals continue this way, are right by the numbers, and found in most introductions but often without mentioning potential shortcomings or controversies and which of these pre-assumptions are not fully proven by now but seem very likely. But could miR-functions maybe differ sometimes or to some extent? Mainstream miR-publishing had begun: a closer look at the evolution of the field and its publishing might be helpful and revealing Casey et al. ${ }^{12}$ These and further first-founding keystone publications and the references herein have suggested canonical miRNA-processing and canonical miR-functions that repress mRNAs which have remained an undoubted cornerstone in the field Guo et 
al. ${ }^{13,2}$ (and references there in) that became somewhat indubitable by peer-pressure-bias. But could they still function differently? Although several important and interesting findings and claims might have been reached and published, they cannot fully resolve all of the potential roles and functions and non-functions of miRNAs without discrepancy. They found interesting roles of miRNAs and apparently brought our understanding a big step further. But maybe not always in a fully correct direction. How much of it is right and fully correct in all of its details?

Due to a bias based evolution of all scientific fields of today, going back has generally become almost impossible by the means of mainstream publishing that follows believes and dominating views and hereby causes subsequent peer-review biases, a major overclaim-bias, and funding biases among others. In this case, mainstream publishing has led to the evolution of the miR-field Casey et al., ${ }^{12}$ one might assume. As a result, the field has much proceeded as if the functions of miRNA and their seed-binding sequences were all now fully identified, and all roles from basic to biomedical became unquestionable in a field that has to steadily deliver anew, which is achieved more easily in the mainstream with add-ons to peer-believes creating more bias in return. Some results might be simply not the case one might assume, but have manifested in the bias of a semi-false self-fulfilling-prophecy and a first-description-publication-bias and a dichotomous bidirectional null-hypothesis-bias that are all in sum very difficult to revert, next to the interesting and important part of the primal findings of this miR-gene function 'screening' of the entire field. It mainly turns problematic, if alternative important hypothesis and projects, if $\mathrm{PhDs}$ and PostDocs are hindered. Such projects are not funded due to this bias, peer beliefs, and overwhelming literature in all Life Science disciplines, Biochemistry and Molecular Biology, Cell Biology, Oncology, Cell Biology, Genetics Heredity, Science Technology, Biotechnology and Applied Microbiology, Research Experimental Medicine, and so on Casey et al., ${ }^{12}$ seemingly generally very much based on adhering to the common believes in all fields. Thereby, strategic bias protects the dominators of the field from falsification and new dogmas, while all other singular and different claims are simultaneously not further verified, recognized or reproduced as peers mainly follow and reinforce the mainstream bias-a double-whammy-bias everywhere.

Subsequently and consequently, miRNAs were already researched for many hundreds ofmillions, in $€$ and $\$$, and published in many countries Casey et al., ${ }^{12}$ mainly in the USA, China, Germany, UK and Italy, in both diagnostics and therapeutics, already much before the basic canonical and non-canonical miR-functions were fully characterized, resolved and entirely understood. This might have been a too premature investment prioritization as one still tends to look to some extent for the wrong things, i.e. wrong read-outs, assuming other basic mechanisms, and biomedical effects. Critiques are often turned around or considered immature if they reveal shortcomings or criticise how funding is done today. Realistic views often don't get the money needed to be mature: maturity seems to correlate with falseness as many kids might have already guessed early in their life. But why would the world demand semi-correct sciences? Early investment in funding of biased R\&D-proposals can only temporarily be beneficial and is in tendency not fully sustainable by accelerating new cherry-pick-based findings, results, patentable ideas and alike, or help to identify promising new things. However, these are maybe not followed up correctly due to the too early stage costs and field biases. Hence, those projects will often be stopped and a follow-up is not possible due to many things like confidentiality agreements of industry research and not publishing of negative results in academia (anti-null-hypothesis bias), or false-failure-bias from previous research. All this early securing R\&D opportunity can be devastating for PostDocs and junior researchers and for the world of science and the biotech industry, especially in the long-run that we are already infor a while as it seems.

One could estimate such miR-discovery projects as "too early and biased" with regard to an analogy of Moore's law of doubling speed in technology that can exponentially shrink costs and focus efforts-not only on the card-, which Moore found for microprocessor transistors initially. The learning curve also plays a crucial role in this doubling speed but only if it learns without false biases. One can maybe compare the miRNA-discovery field with the first sequencing projects of the human genome: 10years later it would have been already extremely more affordable to discover, in fact, and recently especially after 2008 (NIH data). To be first can be important but can also come at a high price - also for the many disadvantaged postdoctoral researchers of today ${ }^{6-8}$ that on top of this have to compete internationally. Today, 10-20years later a new picture emerges in which isomiRs and isoSeeds could also be a key part of the solution, like potentially unforeseen roles. As a direct result of this publication bias and the general biases in the sciences of today, ${ }^{4}$ the field had to research and publishes frequently in this way:

\section{a. Identify miRs in disease e.g. via expression}

b. Find their targets, as a mainstream research strategy

c. Gnerally do not doubt or research the core mechanism again. But there must be more flexibility in research and a new way of thinking and trying out.

To tackle the status quo is risky and in many contexts not allowed, like questioning authority, due to daily training in obedient behavioral every day for most of all people and researchers, unopposed biasing effects already knows since Pavlov and Milgram. Also, the status quo in research, like a real political topic, is risky to criticise still in all countries. Especially in light of senior networking and the systemic oppression of most junior researchers, ${ }^{6-8}$ which seems to work via default static and capriciousness-like dynamic network control of all research projects and research objectives and funding. For most junior researchers, PhDs, and PostDocs, it was not possible to aim for new breakthroughs even if it is clearly the next logical step; who gets the opportunity to work on it defines the success that is built on the ideas of those who did not get the opportunity; top ideas are also immediately reserved and frequently 'transferred' to seemingly privileged researchers. Still, $\mathrm{PhDs}$ and PostDocs always have to be transparent at any times and must present all their new findings in progress reports without the right to publish them. Some senior networks lead into a strategic asymmetric information bias that can harm $\mathrm{PhDs}$ and PostDocs by hindering them, individual per individual, and does so in many $\operatorname{cases}^{6-8}$ and until today-despite all of their excellent work. Seniors in general, when biased by default, often just want or wanted their home-run with a new interesting $\mathrm{miR}$ and a touchdown on a promising target-no matter how-in biasstatic projects by the numbers. No matter which isomiR, no matter the shortness of seeds, no matter the costs to science and for all PostDocs - just key figures and 'something publishable to publish, which could cause a bias. Even if the peer-reviewer is not biased simply assuming that he or she is, or will be, or might be, already very many biases the general author ex ante in many cases. The bias will usually be followed, no matter what the intimidated junior researchers worldwide would say. Blockades in academia, it is difficult to escape into industry, as PostDocs are recently also discriminated on the entire 
job markets due to organized-crime-type strategy consulting firms that strategically bias HR-criteria against PostDocs to keep solutionfinding competitors away from firms by creating artificial scarcity for IQ-jobs in firms; PostDocs would also be the better consultants.

Just in case juniors are still brave enough to say something to the PI or supervisor when a red line of science is once again crossed by the field or lab, generally speaking, they would often risk much by simply mentioning it. We all have to adjust, all of us. But this leads automatically to an adjustment-bias that can be strategically controlled by stimuli from the outside of the lab-and that is what happens. All researchers of these decades, as it seems, tended to be not free of all biases and are somewhat constrained to drive new projects without bias, and biasing pressures, or without further researching a potential bias. ${ }^{4}$ This has led to a biased publication landscape of probably many hundreds or thousands of potentially unreproducibly identified or predicted miRNA-target genes and mechanisms in probably most model organisms, tissues, and cells. So it is obviously difficult to assess how wrong science has actually gone, and how much of it is actually right, what is underrepresented and missing, and which results to believe, and in which way to correctly interpret them to extract the real information again. There are of course also many interesting right findings and results but all leads to the question: Is our understanding significantly shaped by representation bias in all sciences? Nobody can check all this either. To falsify or verify potentially biased results or hypotheses is often a big no-go in most labs due to reasons of cost and time, at same, new hypotheses have it difficult. A system of all hypotheses and alternative hypothesis to verify and falsify is missing. Systems Biology is only a trendy word by now without a further prioritization. It has become difficult to find anything on Pubmed too and a systematic order is missing everywhere. And in the lab, even if a junior or senior researcher spends money and time and reveals a difference to the mainstream publication bias, it will be often not worthwhile and too difficult to publish such news in the widely selectively biased editor-and peer-review community that mainly favors interesting news that must be affirmatively in line with recent believes and biases. To show how it really is or works doesn't get the score. Clearly, nobody checks for all of these bias issues and tries to normalize it. There are not many brave enough to mention them in this network of dependency that profits from bias and from eliminating postdoc martyrs that would otherwise become new big shots and leading top experts- this bias is thus also about the early elimination of scientific competition, and to control all options also in the field of the R\&D industry. Nobody stops such strategic bias developments that start with the position.

Back to the miR-field: subsequently, eye-washing bioinformatics approaches have emerged in the mainstream bias that has generally tried by system and algorithm to reveal and predict miR-targets based on the published miRs-sequence, the published seed sequence, and selected mRNA-targets. miR-Base serves here as both, a helpful but also misleading self-styled gold-standard-like miRNA-database, ${ }^{14}$ a miR-registry that follows its own semi-approximating annotating rules and normalizations that are used by most miR-scientists in the field of today. Especially when it comes to miR-sequence definition, and which is recently widely used in its latest version $21 .^{3}$ There seems to be no right way to pick one miR-sequence, in fact, most if not all miRs is isomiRs. Which isomiRs or research would be the question to be resolved in the first place by scientists? The standard has maybe achieved more comparability but at a too early time point when we still do not know if there is a difference in all isomiRs. The latest version of miR-Base now includes more important deep sequencing data, which was less and totally missing before or more incomplete in earlier years ${ }^{3}$ when the microRNA field and our understanding but also our misunderstanding and miR-bias were just forming. miR-over simplifications might have taken place precipitating in the thinking of the peer network and miR-industry. Our understanding was maybe too early to firmly established with many final views and biases ever since the first publication-when most of the deep sequencing and NGS data was still missing, preliminary or simplified in miR-Base without giving obvious good reason or explanation for most if not all entries and miR-sequences. There is a miR-Base data entry bias and we do not have access to the site that defines the field. All decisions in sciences are now made behind closed doors. As a result of this bias, (I) miRidentity and (II) seed sequences and their target specificity, and (III) the molecular mechanisms have been incompletely assessed. They were often researched without comprising the possibility of a relevance of isomiRs or isoSeeds in the laboratory and in bioinformatics. Weren't they?

\section{miR-identity bias}

The abundance of isomiR-sequences makes it difficult to reveal which of them are the right miRNAs, i.e. isomiRs, that bear a biological function and relevance and so on and if "isomiR-trimming 'could be a relevant new miRNA-mechanisms. Everybody has blindly believed miR-Base and related mainstream biased information donors, and a whole little industry network was designing miR-qPCR-systems, NGS, and chips, for such miRNAs that were all semi-wrongly defined only on the isomiR-MIMAT nt-level, no matter which firm: major life science firms were taking the miR-Base downloadable sequence information, which was called "the gold standard" and wanted to make business this way. Nobody took much care of an isomiR-diversity that is findable today all over the place e.g. in miRSeq data as the products of the genomic miR loci. Subsequently, most researchers were not allowed to doubt anything any longer about it and some lost their jobs if they wanted to use a more precise and differentiated method to validate isomiR expression or function. The research field and miRinnovation industry thought they knew already the facts of the miRsequences and made patents and kits based on a one annotated miRBase sequence but all isomiRs were often not incorporated enough nor was only one sequence to be found as "the-miR-sequence". Until today, these sites misleadingly suggest a reliable identification of miRs based on high coverage and high replicas of deep sequencing reads. ${ }^{3}$ But this high amount of experiments and reads, and reads permillion, can be still misleading and biased if it only suggests one unique miRNA sequence with a MIMAT-ID. Already a brief look through the deep sequencing data reveals a diversity that cannot be simplified so much and so easily in only one entry. Moreover, if you do your own NGS or deep sequencing experiments as a biological and technical replicate you will often find that it only generally resembles these data only as a good overlap of isomiRs or similarity but also with striking differences in quality and quantity. Furthermore, there seems to be no good way chosen to standardize these NGS data results so far on several levels. If one compares different tissues and cell types and miR-methods one will also find isomiR-quantities that will vary even more. This also shows that the normalization must be wrong.

But nucleotides and multiple-nucleotide differences of isomiRs can or could make a big difference, in qPCR-assays as well as in biology-even one nucleotide could in theory. This cloud of nucleotide variation in length and sequence does not only stem from genome and transcript processing, but also from editing, tailing, modifying, trimming, shifting (5' or 3'), substituting and additional mechanisms that lead to this prevalent diversity around one miR-sequence. It spreads like the variations on a common theme around a core overlap 
sequence that does not necessarily and not always represent the unique miR-BASE entry-likely most frequently not. This mass in miR-diversity, i.e. isomiR diversity, is mainly found on both the 5' and 3' strand. Also, the star-strand was initially defined as the opposite strand of the active miR-repressor strand. Subsequently, it was found that this star-strand could also exert a repressive activity - again indicating an incomplete and biased understanding in the miR-field that has manifested to early. For instance, if strands can switch-why should seeds not be able to switch-which is now termed isoSeeds, i.e. seed variations per miR that could differently match a mRNA-target, to have a term for it. The number of isomiRs and their diversity will be even higher from tissue to tissue and cell type to cell type, and miRBASE standardization ${ }^{3}$ will be even more difficult and semi-falsely normalized. This makes miR-Base still a great helpful tool but also a misleading one at the same time, and the last feature is the problem. Conserved elements stay the same but striking amounts of variants cannot be simply omitted by simplifying into one unique MIMAT-ID sequence. But exactly this has been done and was taken as a given by many in the research field and also by the industry. It has helped to sell the first generation of miRNA-qPCR-kits that PhDs and PostDocs had to use without being allowed to question their meaningfulness in light of ubiquitous isomiR abundance everywhere in all tissues and cells for most miRs with important variations and diversity in most of all cases. QPCR-data are often used to validate the expression of chip or NGS data - but most qPCR is very nt-specific and very ntsensitive but in a very different way than NGS and chip methods. Thus, isomiRs can interfere with validation via both specificity and non-specificity, especially if they are the most abundant miRs in the sample. Hence, validation of miR-expression via $\mathrm{qPCR}$ can be hampered by a higher isomiR-abundance and leads to a bias already on the molecular method level, the same holds true for chip and NGS data methods but differently.

Faithful, most projects followed all biases and shifted the entire field into a zone of miR-identity, validation bias and functional bias, which was all the beginning and core of many projects. My postdoctoral qPCR miR-validation data could also show that isomiRs affect the validation rate but I was not allowed to publish these important methodological findings, which would have questioned the utility of qPCR validation kits. Like with most of my key projects I was hindered to complete and publish them as a PostDoc (hence, data not shown), starting with the confidentiality agreements that most PhDs and PostDocs must sign these days. In summary, whenever isomiR-abundance was high the qPCR-validation results tended to be less significant e.g. when aiming at a qPCR-validation of a chip-result, and in comparison with independent NGS data too. Still, this is the tandem method of validation in use which is found in many miRNApublications, and isomiR-abundance is often neglected due to costs and time. Understandably, but this can be contra-productive, as the goal remains to identify the real isomiRs that bear the biological role and the relevant expression-and to understand if there might be often differences of isomiRs-which would make them more important. In summary, to understand the effects of miRNA expression one cannot speak of one miRNA or one of two miRNA strands-one should keep in mind that it is a cloud of isomiRs and often not one sequence. The relevant miR might be a different isomiR and actually, all isomiRs must be validated at least high-level one, which is still too costly today. Better validation methods for isomiRs can be innovated (methods not shown). Quantity comes into play, but as validation still partially fails it is only a first semi-quantitative guess that seems to be grounded on biases and remains isomiR-, context-, tissue-, und method-specific. Importantly, most graphical and statistical representations, also on miR-BASE might falsely imply an unbiased and standardized representation of isomiR-abundance, which is always biased but to which extent? MIMIAT-sequences are widely taken too early as the final finding by researchers and method innovators. Disturbances of molecular quantification methods by isomiR abundance have not been checked and were not allowed to be checked rigorously. Finally, miRidentity is a matter of isomiR-quantity and quality and a pool of miRvariations is to be studied and not one miR, which would be to cost and time intensive if it is done too early.

\section{miRNA-seed sequences and specificity bias}

Most and major parts of the isomiRs are constant, often evolutionarily conserved, and famous miRNA-researchers have early on claimed a so-called "seed sequence" of 6 to $8 \mathrm{nt}$ that is found 5 " of the miRISC-engaged miR-strand. This seed is usually defined from nt-position 2 to 8 . These canonical miR-seeds were easier to identify due to several reasons: they have a simple evolutionarily conserved continuous seed core that could be easily found in the less conserved 3'-UTR counterparts of mRNA transcripts. Binding of miRISC-the miRNA-induced silencing complex-is intended to block translation or to represses and reduces transcripts via transcript stability and halflife values $\mathrm{t} 1 / 2$ supposedly by polyadenylation, decapping and 5'-to-3' exonucleolytic decay. ${ }^{2}$ This idea initially goes back to the previously discovered siRNA mechanisms and reports that many miR-seeds were identified with striking complementarity to mRNA transcripts. ${ }^{15}$

This is, however, just a matter of statistics in genomics. In other words, even if there were not one real target of a specific miRNA you would always find tens of thousands of potential false-positive targets due to the shortness of the seed sequences: 1 nucleotide makes 4,2 gives rise to 16,6 gives rise to $4^{\wedge} 6$ which is 4096 a very low number in comparison to the human genome of 3,2 billion or the human transcriptome of ca. 32million base-pairs, approximately $1 \%$ of the genome, which makes roughly $4^{\wedge} 32 \mathrm{Mb}=10^{\wedge} 10^{\wedge} 75^{\prime}$-to3' directional mRNA-sequences possible. Like a restriction enzyme that cuts a $3+3=6 \mathrm{nt}$ palindrome, the miR-seed would also statistically bind every 4096 nucleotides on average-given randomness. Thus, the transcriptome has 7800 times more sequences in length than needed to find a $6 \mathrm{bp}$-seed match. One miRNA with a 6-bp-seed would already match with thousands of targets (7800), which is unlikely to ever result in a specific effect of a miRNA that could have evolved over time. Hence, where does a higher specificity arise?

Even a long 8-nt-miR-isoSeed that binds a target mRNA with 8 nts would still bind to every 65536 random nts: 488 times in the transcriptome. The question of specificity arises but was quickly set aside again after the papers of the Bartel laboratory came out. ${ }^{15}$ To achieve hundreds of mRNA target genes it would require a seed of 8 conserved canonical core-seed nts, which is already relatively long compared to publications of specific miRNA effects on PubMed 2017. Hence, it was claimed that seed length correlates with efficacy ${ }^{1}$ but not always. Single-stranded RNA is more flexible than double-stranded DNA and this further increases the binding likelihood and would put even more emphasis on this reoccurrence of an old question: How can a miRNA-mRNA target-specificity arise and is there a hidden role of miRs on genomic DNA and protein-interactions? Not every potential transcription factor binding site binds a transcription factor in the genome. But base-pairing can be more sequence-specific than most protein-DNA or protein-RNA interactions. RNA behaves different structurally than DNA. RNA and DNA are nearly identical: RNA has a ribose and DNA a desoxyribose making it more rigid and a uracil instead of a thymine base. As a result, dsDNA is a B-helix 
and dsRNA is a compact A-form under physiological conditions - this could allow proteins to structurally discern between the two-and the single-stranded versions are structurally very flexible and can bind via Watson-Crick and non-Watson-Crick-base-pairing. RNA resides mainly as single-stranded RNA or RNA stretches with loops, with specific and also dynamic binding affinity and binding flexibilityhelix-parts form just thermodynamically more stable sub-forms: imperfect miR-helices might give structural codes and cues.

During miR-biogenesis a pri-miRNA is transcribed from the genome and forms single-stranded stem-loop structures that are thought to be processed by a Drosha-and DGCR8-containing microprocessor complex (1:1 and 1:2 stoichiometry?) into the shorter pre-miR stemloop semi-helix structures. They are exported into the cytoplasm (Exportin-5/Ran-GDP) where most of them are further processed by the RNAase-III enzyme Dicer into classical miRNAs. Factors like TRBP2 could help Dicer to cleave the miRNA stem-loop and in the next step the Ago2/miRISC complex is recruited Chendrimada et al. ${ }^{16}$ to regulate mRNA inhibition, all these mechanisms were probably claimed and established a bit too fast and thus could be significantly biased.

\section{The mRNA transcript repression is believed to be mediated by}

i. mRNA transcript stability and induced degradation in a siRNA-like mechanism.

ii. Translational repression by somehow blocking ribosomes and that both could be an inevitable interplay. ${ }^{1,2}$

It has been suggested that mammalian microRNAs predominantly ( $>84 \%$ ) act to decrease target mRNA levels via RNA silencing Guo et al. ${ }^{13}$ using ribosome profiling-but the numbers could be 'biased' and still different. Conserved miRNA sites in 3'-UTR could, in fact, lead to a higher mRNA destabilization while the open reading frame (ORF) could also block translation. These interesting assays Guo et al., ${ }^{13}$ however, cannot confirm the exact quantitative level of overall ribosomal repression, which could vary between transcripts and is always dynamic like ribosome speed and not static like RPFs (ribosome protected RNA fragments). Many miR-target effects could be artificially measures due to the ectopic, overexpression, and other methods used and different mechanisms might still be in play, which was neither publishable nor researchable due to the imperative to stick with the predominant and biased views and peer-pressures.

With respect to isomiR and specificity, cooperativity of the official miRNA and all of its isomiRs has been suggested Cloonan et al. ${ }^{17}$ A closer look at the details of the miRNA-publications, however, reveals, that most bioinformatics predictions mainly fail due to bias and predominantly result in semi-false or misleading predictions (www.mirdb.org, www.microrna.org, www.targetscan.org, and all the others too). No one in this field of science seems to be able to fully assess the truth value of these predictions accordingly and correctly but everyone takes this bias as an arbitrary given. PostDocs are hindered via hierarchy and publishing criteria to reveal the truth-value about these predictions. Biochemical experiments and HITS-CLIP Moore et al. ${ }^{18}$ sees a striking overlap of these $6-8 \mathrm{bp}$ bioinformatics predictions for miRs, also according to research talks on conferences, but this seems unlikely to be the case in light of the fact that most isomiRs and isoSeed are not well defined. That HITS-CLIP and bioinformatics predictions Chi et al. ${ }^{19}$ overlap so strikingly can have different reasons, and could bear a wishful-thinking bias: e.g. the biochemical results are only "ago-footprints" and all models stem from lets-find-the-correctseed-matches in miR-and mRNA-datasets. Who says that all seed and miR-target prediction are correct? Probably nobody and more and more recent papers have mentioned correctly that bioinformatics went very wrong and misleading. Bioinformatics is helpful but its downsides and misleading biases bear many problems. They ideally summarize our misunderstanding too next to our biased knowledge. This bioinformatics-based-bias is also found in salary, why should bioinformatics PostDocs earn more than laboratory PostDocs? There is actually no scientific reason for this and must go back to an informatics-driven 'strategy consulting network' based discrimination of Life Science PostDocs and PhDs internationally via strategic HR bias: strategic job requirement bias in academia and industry. For instance, only professional and transferable PostDocs-experience and competency is intentionally viewed negatively by a false advisory strategy, all other job experiences count always very much. Who else but a consultancy could do such a crime to science globally?

\section{Functions of miRNAs Bias}

The miRNA-field has maybe rushed into its established views and mechanism a bit too fast-in a time of untimely breakthrough discoveries. Subsequently, nobody could claim anything else in a biasedly semi-dysfunctional peer-review community. Although this has led to many interesting primary and secondary findings, it has also created a new big obstacle to publish in an unbiased way. Maybe as much as it has become a big obstacle to know what is true and what is not of all that has been published under the peer-hierarchyand network-pressures and the imperative to publish, publish, publish-irrespective of the overall truth value (OTV), the true novelty value (TNV), the level of bias (LOB) and the real value of scientific achievement (RVSA).

Next to inhibition of mRNA stability and translation ${ }^{1,2}$ miRNAs could potentially have many additional but undiscovered functions due to the bias: some could also

i. Have only minimal effects as RNA degradation products,

ii. Still play a role on DNA and in epigenetics, genomics and transcription,

iii. Fine-tune protein-stability and function; activate functions of different proteins

iv. Drive and change protein-DNA, protein-RNA, and proteinprotein interactions,

v. Act indirectly on targets mRNAs i.e. via signaling pathways and alike Anton et al., ${ }^{20}$ and

vi. Control molecular mechanisms as other classes if they are inseparable from miR-definitions by sequence: like passenger-, sno-and guide-miRs

vii. Be cellular sequence-binders for other mechanisms like editing, mRNA decay, and alike,

viii. Contribute toward cellular-immunity and health, anti-pathogen defense

ix. Mediate robustness or quality of cell fate, transcription networks, cell cycle, communication, homeostasis, and other mechanisms

x. They could still have unforeseen additional functions that are not investigated due the massive bias that only few contest.

The methods that quantify expression are technically biased and yield different and contradicting results, like the methods that analyze 
the biological role and function of miRs. For example, qPCR vs. Chip vs NGS. Additionally, biological overexpression and silencing studies have all yielded data that are frequently at odds with loss of function knock-out (KO) studies. ${ }^{21} \mathrm{KO}$ studies thereby also reveal more dispensability ${ }^{21}$ than was previously expected, due to reason that authors do not submit negative result in light of a peer-review journalism bias and anti-null-hypothesis-bias, and high-impact editors that are not highly interested in no change figures or negative results, although they might be invaluable and worth like gold for future funding. There is usually also more time between the experimental manipulation and measurement that allows the biological system to recover from the intervention or it might also override the real biological relevance if there is any-general method bias.

A screen of miRNAs regulating a signaling pathway preliminarily yielded miRNA classes the either up or downregulate transcriptional activity as measured by a specific TCF reporter Anton et al. ${ }^{20}$ An alignment of these miRs reveals that the functional miRNA sequence similarities are high in the functional classes, i.e. repressor and activator, often bear a striking sequence similarity. Conserved sequence similarity comprises more than $6-8 \mathrm{bp}$ seed and could bear patchy non-canonical seeds throughout the miR-sequence. ${ }^{20}$ On average, findable sequence similarity is $14 \mathrm{bp}(\sigma=3 \mathrm{bp})$ in the top hits group of miRs. If this sequence similarity reflects a non-canonical seed remains to be proven, but it could yield a much higher specificity that was observed for the pathway: $4^{\wedge} 14$ equals 268 million that would yield 1 miRNA for $1 \mathrm{mRNA}$, if such discontinuous seed matches exist and if they are functioning this way. Even a discontinuous $12 \mathrm{nt}$ seed could achieve this effect:

i. $\mathrm{miR}$ for ca.

ii. MRNAs assuming randomness-and there might be noncanonical Non-Watson-Crick specificity arising too. This all could explain a higher level of specificity of miRs via isoSeeds and non-canonical seeds (Figure 1).

The presence of isomiR-diversity with 5 'shifts in NGS data makes non-canonical miR-seed very likely as the start positions are already this way shifted away from the canonical position 2-8 (Figure 1).
In opposite to canonical miRs and miR-seeds, non-canonical miRs are isomiRs that can form various seed structures that might differ from target to target, termed isoSeeds. Both defining features are summarized in Figure 1. Today, there is a plethora of literature bias towards canonical seeds-and it will be interesting to elucidate noncanonical and isoSeed functions in the future. But this has been and will be more difficult to reveal due to the often forgotten level-ofdifficulty-bias to verify, as such non-canonical seeds would bind in a less predictable discontinuous way. To be less stringent and less canonical in all key miR-definitions could open entirely new routes for potentially important miR-research on non-canonical types and for new R\&D-ideas. We are all biased but how biased are we really? What is the real level of bias, or LOB? Do we have to accept that most research findings are wrong and semi-right? ${ }^{4,22}$, Do we need to always accept the extreme peer-pressure bias of today stemming from an organized network that could bully us and affects and controls our employment and the stereotypes that it publishes? Yes, the LOB is generally not researched and not known by the senior network community and there is no junior impact, but it should be in fact always assessed, as most research finding could be, in fact, wrong due to all of these biases. ${ }^{4,22,23}$ How biased are we in this specific miRNAfield? Are all previous reports of miRNA-repression examples of unspecific effects and only a somewhat global down regulation due the methods used? Can the canonical 6-8nt seed sequences explain all of such biological effects? How strong are our miR-identity-bias and miR-function-bias? Maybe not all that strong but also not that weak. There could be both, more and less specific miRNAs at the same time, with roles and without roles, with different seed structures, isoSeeds, and isomiRs. Could it be that a historic publication bias and its imperative has given us prefabricated views and false preassumptions for only their own success? Yes, and did we overlook additional factors, routes, mechanisms, and explanations? Likely, and do we too early agree with dominating authors in the field? Well, the peer-review community does so, excludes different opinions and restricts all junior-only contributions by eligibility and publishing costs, and is known to benefit established authors, hence all readers, all of us, are much biased in sum and irrespective of important work that 'big shots' can do or might also have done.
A

Canonical miR-Seeds

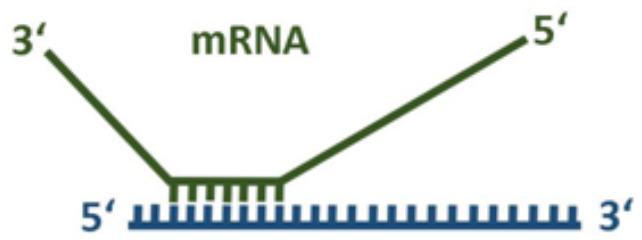

length 6-8 nt

conventional

5'-seed: position 2-8

Watson-Crick-base-pairs (bp)

continuous

conserved stretch

maybe more UTRs than ORFs

transcripts and translation

shown with bias

\section{B Non-Canonical miR-Seeds}

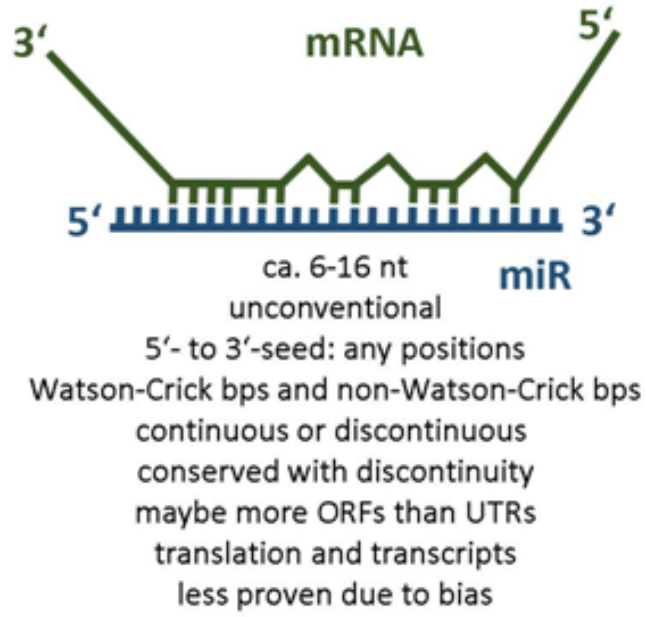

Figure I Canonical and non-canonical mir-isoseeds, specificity via unconventional basepairs matching? 


\section{Conclusion}

Despite the false pre-assumptions in the field about (a) only one unique miR-sequences instead of pools of isomiRs, of (b) only one seed instead of multiple isoSeeds, of (c) only one main function instead of many functions, of (d) only one often false positive and false negative bioinformatics approach instead of promising ideas and new algorithms and ways to potentially find the right targets, the field still has-after all (a-d)-identified interesting key mechanisms and many miRNAs with a potential biological relevance and biomedical impact. However, how many of these biomedical miRs that were identified in this cherry-picking approach of the science field can be verified biomedical and promising targets and candidates for therapeutic and diagnostic strategies remains to be reinvestigated due to the obvious biases and pressures in science of today. ${ }^{4,22,23}$ There is a too high pressure on most of all junior scientists, on $\mathrm{PhD}$ and PostDocs who are the real scientists in the lab who do the research, and also PIs stand under high peer bias pressure to obtain funding and kudos. Sustainable career paths are lacking like good jobs and PostDocs are discriminated due to consultancies in all suitable jobs. This increases the bias and peer pressure in science and nobody 'quality assures science' or assures fairness for juniors and protects it from the biases of peer-review, funding and publication biases. In order to take the next big step and to take the field to the next level, it will be important to overcome the biases in science..$^{4,22,23}$ This includes a correct view of the situation of all PhDs and PostDocs and to assure sustainable career paths.

With respect to the miR-field: Scientifically, it might be interesting to reveal

a. If there are different types of seed sequences that are biologically relevant: canonical versus non-canonical seeds, and multiple seeds termed isoseeds.

b. If there are different types of isomiRs that are biological relevant: canonical and non-canonical isomiRs.

c. The real or additional mechanisms involved.

d. How to improve our methods to tackles all of these questions and molecular heterogeneity, complexity and diversity of isomiRs an isoSeeds.

Recently, biomedical researchers and bio-informaticians have also correctly realized this new challenge of isomiR complexity and are eventually working on this non-canonical isomiR topic Li et al. ${ }^{24}$ The same could be done for the non-canonical seeds, and the correct algorithm to align them with mRNAs has still yet to be identified, or the old algorithms have to be systematically re-investigated and proven alternatively. Canonical miRs and miR-seeds were easier to identify, but the majority of miRs could be non-canonical depending on the cut-off of the definition which is set to $2-8 \mathrm{bp}$ for canonical seeds due to historical reason. Most publications highly intend to give the strong impression of science but are highly biased and pseudo-scientific. Everyone who criticizes them is subsequently called this way by a dominant peer pressure bias. Many rules to avoid bias are everywhere often not followed at all. ${ }^{4,22,23}$ Senior researchers and peers have also intentionally introduced new strategic biases of great implications and falseness to omit the underlying complexity and diversity and to mainly block the success of all competing researchers, to publish easier, or to make their career, or to sell products, like the miRNA qPCR kits that are biased by the MIMAT ID norms, or to silence all junior and competitor researchers to gain an advantage and more time at the cost for all and for science. Only if we research biases we can avoid them. Only a scientific system that works in theory can work in practice. Systems Biology only works if we are doing it togetherwithout excluding or repressing scientists.

\section{Acknowledgements}

I acknowledge University of Truth International to support me in this work that was previously censored by PIs and peers and the research system by strategically blocking most postdoctoral reports and careers.

\section{Conflicts of interest}

Conflicts of interests are prevalent in all research of today due to the wrong hiring practices. A sustainable research system without such conflicts of interests is needed. All publications of today that do not mention these conflicts of interest from peer-pressures and from what is needed for success still omit the full truth. The University of Truth comparable to School of Life did not financially support me in any way, nor did anyone else benefit me for this review work. I did also not receive any relieve, which in sum helped me to be harshly compelled to avoid any potential financial bias or dependency that could inflict the unbiased nature, independence, and outcome of this work since every remuneration and financial support would anyhow always result in a never confessed conflict of interest and bias. Future job prospects were set aside only for the time of writing this review. I will have to comply with all biases to be compatible again.

\section{References}

1. Bartel D. MicroRNAs: Target Recognition and Regulatory Functions. Cell. 2009;136(2):215-233.

2. Jonas S, Izaurralde E. Towards a molecular understanding of microRNAmediated gene silencing. Nature Reviews Genetics. 2015;16(7):421-433.

3. Kozomara A, Griffiths-Jones S. miRBase: annotating high confidence microRNAs using deepsequencing data. Nucleic Acids Res. 2014;42(D1):D68-D73.

4. Rothstein H, Sutton AJ, Borenstein M. Publication Bias in MetaAnalysis-Prevention, Assessment and Adjustments. Chichester, England: Sutton and Borenstein, John Wiley \& Sons; 2005.

5. Shinbrot T. Exploitation of junior scientists must end. Nature. 1999;521(399):521.

6. Bourne HR. A fair deal for $\mathrm{PhD}$ students and postdocs. Elife. 2013;1(2):e01139.

7. Holleman W, Gritz ER. Biomedical burnout. Nature. 2013;500:613-614.

8. Alberts B, Kirschner MW, Tilghman S, et al. Rescuing US Biomedical Research From Its Systemic Flaws. Proc Natl Acad Sci USA. 2014;111(16):5773-5777.

9. McDowell GS, Gunsalus KT, MacKellar DC, et al. Shaping the Future of Research: a perspective from junior scientists. F1000Res. 2015;3:291.

10. Lee C, Feinbaum R, Ambros V. The C. elegans heterochronic gene lin4 encodes small RNAs with antisense complementarity to lin-14. Cell. 1993;75(5):843-854.

11. Bartel DP. MicroRNAs: genomics, biogenesis, mechanism, and function Cell. 2004;116(2):281-297.

12. Casey MC, Kerin MJ, Brown JA, et al. Evolution of a research field-a micro (RNA) example. Peer J. 2015;3:se829.

13. Guo $\mathrm{H}$, Ingolia $\mathrm{N}$, Weissman $\mathrm{J}$, et al. Mammalian microRNAs predominantly act to decrease target mRNA levels. Nature. 2010;466(7308):835-840. 
14. Griffiths-Jones S. The microRNA Registry. Nucleic Acids Res. 2004;32(Database Issue):D109-D111.

15. Lewis B, Burge C, Bartel D. Conserved seed pairing, often flanked by adenosines, indicates that thousands of human genes are microRNA targets. Cell. 2005;120(1):15-20.

16. Chendrimada TP, Gregory RI, Kumaraswamy E, et al. TRBP recruits the Dicer complex to Ago2 for microRNA processing and gene silencing. Nature. 2005;436(7051):740-744.

17. Cloonan N, Wani S, Xu Q, et al. MicroRNAs and their isomiRs function cooperatively to target common biological pathways. Genome biol. 2011;12(12):R126.

18. Moore MJ, Zhang C, Gantman EC, et al. Mapping Argonaute and conventional RNA-binding protein interactions with RNA at singlenucleotide resolution using HITS-CLIP and CIMS analysis. Nat Protoc. 2014;9(2):263-293
19. Chi SW, Zang JB, Mele A, et al. Ago HITS-CLIP decodes miRNAmRNA interaction maps. Nature. 2010;460(7254):479-486.

20. Anton R, Chatterjee SS, Simundza J, et al. A Systematic Screen for Micro-RNAs Regulating the Canonical Wnt Pathway. PLoS One. 2011;6(10):e26257.

21. Joana A, Ventura A. The biological functions of miRNAs: lessons from in vivo studies. Trends in Cell Biol. 2015;25(3):137-147.

22. Ioannidis JPA. Why most published research findings are false. PLoS medicine. 2005;2(8):e124.

23. Pannucci CJ, Wilkins EG. Identifying and Avoiding Bias in Research Plast Reconst Surg. 2011;126(2):619-625.

24. Li G, Jiafeng Y, Liang T, et al. miR-isomiRExp: a web-server for the analysis of expression of miRNA at the miRNA/isomiR levels. Sci Rep. 2016;6:1-7. 